\section{RADIO COMMUNICATION DEVELOPMENTS}

$\mathrm{A}^{\mathrm{T}}$

$T$ the inaugural meeting on October 10 of the Radio Section of the Institution of Electrical Engineers, the chairman for the present session, Mr. A. H. Mumford, gave an address in which he reviewed some of the more general communication developments carried out by the Post Office Engineering Department during the past few years. These developments covered three main fields : first, the extension and improvement of the facilities for world-wide telegraphic communication on a low frequency; secondly, the study and improvement of longdistance high-frequency telephone and telegraph circuits; and thirdly, the extension of very-high frequency radio links for multi-channel communication over short distances.

Shortly after the occupation of western Europe in 1940, plans were made for setting up a new station operating on a very-low frequency to act as reserve in case of damage to the telegraph transmitter at Rugby, which has been in operation for some twenty years on a frequency of $16 \mathrm{kc} . \mathrm{s}$. The new station was built on a plain in the west of England, and the aerial is supported by three 600 -ft. self-supporting towers on one side, while on the other the aerial triatics are anchored to the top of a hill some $800 \mathrm{ft}$. high, and with a steeply sloping side to the plain below. The aerial tuning-coil is similar in design to that used in the original Rugby station; it comprises five hexagonal coils, nearly $16 \mathrm{ft}$. in diameter, wound on a framework of American whitewood, the overall diameter of the "litzendraht wire" used being about 1.5 in. The aerial and intermediate circuit inductors are housed in a room the walls and roof of which contain a certain amount of steel reinforcement. By suitably screening the room with copper wire, the losses due to the presence of the steel were decreased considerably, with a resulting improvement in the overall efficiency of the station.

During March 1943, the original radio station at Rugby was seriously damaged by fire, and the main aerial tuning coil was destroyed. Although the reserve transmitter referred to above was nearly completed, it was still deemed essential to restore the original station to working order. Lack of the appropriate materials precluded the reconstruction of the coil to the original design, and accordingly an outdoor aerial tuning coil was designed and constructed. This external coil is of a helical hexagon shape, of maximum diameter $65 \mathrm{ft}$., and is wound with ten turns of a cage of copper wires. The coil is supported by poles $67 \mathrm{ft}$. high erected in a circle of more than $100 \mathrm{ft}$. in diameter. A model of this coil and also of the aerial system at the new station were displayed at the meeting.

Mr. Mumford next referred to some of the difficulties which are encountered in the transmission of high-frequency radio waves (for example, $20 \mathrm{Mc} / \mathrm{s}$.) over a path such as that between New York and London, due to selective fading caused by different frequency components of the signal having traversed paths of appreciably different length. A demonstration was given of some laboratory equipment which simulated the transmission of radio waves over three paths of different attenuation and time delay; by suitably adjusting the characteristics of the three paths, interference and fading phenomena can be reproduced and studied.
With this equipment, it was shown that the distortion of the signal in double side-band transmission due to the selective fading of the carrier wave relative to the side-bands can be appreciably reduced by the use of single side-band technique. Such an improvement has been borne out in practice, and the laboratory equipment using an artificial propagation medium enables the radio engineor to obtain quantitative results which can be immediately applied by the receiver designer. The same equipment can be used to investigate the effectiveness of diversity reception in reducing the distortion of signals, and the extent to which the aerial spacing is critical in such reception.

For the reception of signals from a station in New Jersey, U.S.A., on five frequencies between 5 and $20 \mathrm{Mc} . / \mathrm{s}$., the multiple-unit steerable antenna ('musa') system installed by the Post Office at Cooling, Kent, has been in commercial operation since July 1942. This system comprises sixteen rhombic antennæ spaced at regular intervals over a distance of two miles along the great circle towards the transmitting station at New Jersey. By combining the signals from the individual antennæ with suitable phase shift, the major lobe for reception can be varied in the vertical plane. The system is equipped and calibrated to measure the arrival angles, relative to the ground, of the components of the incoming signal, and their field strengths. Such measurements of field strength and wave angle are made day by day at hourly and quarter-hourly intervals respectively, and the results of the measurements, which are available from July 1942 to the present time, provide data of particular interest to the ionospheric physicist and to the radio engineer. During the address, the signals from America received at the 'musa' station at Cooling were relayed to the Institution and displayed on cathode ray oscilloscopes set up in the lecture theatre.

During the past eight years multi-channel radio links operating on the very-high frequencies between 40 and $100 \mathrm{Mc}$./s. have been developed to form part of the main trunk telephone network to bridge gaps over river estuaries or between islands and the mainland. These links carry twelve speech circuits occupying the frequency band $12-60 \mathrm{kc} . / \mathrm{s}$., which can be conveniently transmitted over a coaxial cable upon which modern trunk communications are based. This development is in fact indicative of the merging of line and radio techniques which is so essential to the effective exploitation of the communication art on both a national and international basis. The earlier installations of this twelve-channel radio link used transmitter powers of 100 watts and amplitude modulation; and the sending and receiving equipment was installed in one building which is normally left unattended. More recently, a comprehensive series of field tests has shown that by using frequency modulation, a number of technical limitations could be overcome and the same grade of service could be provided with a transmitter power of 10 watts. This reduction in power is of vital importance to the unattended operation aspect, since both power supply requirements and bulk of equipment are appreciably reduced.

Later experiments have shown that it is quite practicable to have very-high frequency radio links operating on frequency modulation technique and carrying sixty speech circuits simultaneously, thus allowing an even more complete merging of line and radio techniques. 